„Ihr gelegentliches Zaudern, ihre vorübergehende Ratlosigkeit, ihre Versuche, es möglichst vielen möglichst recht zu machen (...), müssen zu einem nicht unerheblichen Teil als Reaktion auf die dramatisch gewachsenen Anforderungen des Regierens gedeutet werden." (S. 596) Die mediale Dauerbeobachtung des „real-time Journalismus“ (S. 594) und eine Bürgergesellschaft, die gesteigerten Wert auf demokratische Mitsprache legt, wirken wie Bremsscheiben in einem ansonsten beschleunigten Politikzyklus.

In Abgrenzung zur Geschichtswissenschaft, so die These Klaus von Beymes in seinem hervorragenden Beitrag, kennzeichnet die heutige Politikwissenschaft eine „Schnelllebigkeit des Faches“" (S. 156). Das mag zum einen an den sich rasch wandelnden wissenschaftlichen Moden liegen, zum anderen jedoch auch an der oftmals kurzatmig und hektisch agierenden Politik selbst. Um in dieser Ereignisgeschwindigkeit innezuhalten und an den mannigfachen Stromschnellen nicht unterzugehen, bietet die vorliegende Festschrift eine Fülle hilfreicher Ankerpunkte. Die Unterschiedlichkeit der versammelten Kontroversen illustriert nicht nur die Fähigkeit des Landes zur politischen Selbstkorrektur seiner Maßstäbe. Klar wird auch, welche Spannweite an Themen die Politikwissenschaft in den zurückliegenden Jahrzehnten abgedeckt hat und wie sehr ihre Entwicklung mit jeweils aktuellen politischen Veränderungen verknüpft gewesen ist.

Helge F. Jani

\title{
Die Schweiz: vom Extremtyp zum Normaltyp der Konsensusdemokratie?
}

Vatter, Adrian: Das politische System der Schweiz (Reihe "Studienkurs Politikwissenschaft"), Nomos Verlagsgesellschaft, Baden-Baden 2014, 589 Seiten, € 29,99.

Im Februar 2014 war eine eidgenössische Volksinitiative „Gegen Masseneinwanderung“ mit 50,3 Prozent erfolgreich. Bis auf die Schweizerische Volkspartei (SVP) mit ihrem Volkstribun Christoph Blocher lehnten alle anderen Parlamentsparteien diese Initiative ab. Adrian Vatter konnte das Votum, das in der in- und ausländischen Öffentlichkeit heftige Reaktionen auslöste, nicht mehr berücksichtigen, deutet aber die Probleme der Einheimischen mit „Fremden“ an. Sein auf zahlreichen Vorstudien basierendes Buch zum politischen System der Schweiz ist die zweite größere einschlägige Monographie von wissenschaftlichem Rang neben der Wolf Linders. ${ }^{1}$

Das ausgezeichnete Werk, gut strukturiert, perspektivenreich gestaltet, materialreich komponiert, anschaulich geschrieben, mit zahlreichen Tabellen und Abbildungen versehen, besticht durch Systematik. Die Einleitung präsentiert unter anderem den Forschungsstand, allerdings knapp, ferner einen Überblick zum politischen System sowie die konzeptionelle und die theoretische Grundlage. Die Konzeption lehnt sich an Wolfgang Merkels „eingebettete Demokratie“ mit ihren fünf Elementen an (demokratisches Wahlregime, politische Partizipationsrechte, effektive Regierungsgewalt, Gewaltenkontrolle, bürgerliche Freiheitsrechte), die Theorie an Arend Lijphart, der zehn Merkmale für seine Typologie von Mehrheits- und Konsensdemokratie heranzieht. Anders als bei diesem fehlt die Zentralbank, an

1 Vgl. Wolf Linder, Schweizerische Demokratie. Institutionen - Prozesse - Perspektiven, 3., vollständig überarbeitete und aktualisierte Auflage, Bern u.a. 2012. 
deren Stelle die „direkte Demokratie“ tritt. Ob stark ausgebaute Volksrechte allerdings immer ein Zeichen einer Konsensusdemokratie sind? Bekanntlich gilt die Schweiz als der Musterfall einer solchen (im Gegensatz zum 19. Jahrhundert), als Modell des „gütlichen Einvernehmens“. In der von der Vereinigten Bundesversammlung, dem Nationalrat und dem Ständerat gewählten Regierung, dem Bundesrat, sitzen sieben gleichberechtigte Mitglieder. Auch Vatter kann dessen Willensbildungsprozess hinter „verschlossenen Türen“ naturgemäß nicht erhellen. Nach der „Zauberformel“ von 1959 erhielten die Freisinnig-Demokratische Partei (FDP), die Christlichdemokratische Volkspartei (CVP) und die Sozialdemokratische Partei (SPS) zwei Sitze, die vierte, der Vorgänger der SVP, bekam einen. 2003 verlor die CVP einen Sitz an die stärkste politische Kraft, die SVP, später diese einen an die Bürgerlich-Demokratische Partei (BDP).

Das Schlusskapitel ordnet die Eidgenossenschaft vergleichend ein: intertemporal, international, subnational. Wie Vatter in einem „Zwiebelschalenprinzip“ (S. 519) erhellt, haben sich die äußeren Demokratiestrukturen, die inneren Entscheidungs- und Machtstrukturen sowie die Positionen der einzelnen Akteure gewandelt. Der Autor erkennt bei der horizontalen Dimension der Machtteilung eine „Abkehr vom Extremtyp hin zum Normalfall einer Konsensdemokratie“ (S. 527), etwa durch den auf Konflikt angelegten Politikstil der SVP, bei der vertikalen Dimension hingegen Kontinuität. Die Demokratiequalität ist gefestigt, allerdings leicht rückläufig - das Land nimmt gleichwohl weiterhin einen der Spitzenplätze ein. Im subnationalen Vergleich stehen die frankophonen Kantone bei der „liberalen“ Demokratiequalität gut da (den garantierten Freiheitsrechten), weniger gut bei der „,radikalen“ (den plebiszitären Elementen), vice versa gilt das für die Kantone der Deutschschweiz.

Zwischen der Einleitung und dem Resümee sind die Kapitel an Lijpharts Typologiemerkmalen angelehnt: Wahlsystem, Parteien und Parteiensystem, Verbände und Verbandssystem, Regierung, Zweikammersystem, direkte Demokratie, Verfassung, Föderalismus, Justiz. Das Fehlen solch zentraler Themen wie Extremismus, Medien, Politische Kultur, Sozial- und Wirtschaftsordnung sowie der Politikfelder erklärt sich mit diesem Ansatz, den Vatter bereits vor Jahren erfolgreich angewandt hat. ${ }^{2}$ Jedes Kapitel ist prinzipiell gleich aufgebaut. Nach einem einleitenden Abschnitt folgen historische Grundlagen, die Funktionsweise, ein internationaler Vergleich, die kantonale Ebene, Zusammenfassung und Diskussion, Literaturverzeichnis sowie, typisch für ein Lehrbuch, ein kurzer Fragenkatalog. Dieses Vorgehen ermöglicht instruktive Vergleiche. Herausragend sind die auf originärer Forschung basierenden Abschnitte über die 26 Kantone $^{3}$, etwa die Passagen zu den dort noch stärker verankerten Referenden.

Was nicht verwundert: Das für die Schweiz charakteristische Element der „direkten Demokratie“ nimmt den größten Platz ein. Zwar sichert ihre Existenz eher den Status quo, weil im Parlament Konsensfindung angestrebt ist, um keine Niederlage bei Referenden zu provozieren. Doch gibt es in jüngerer Zeit polarisierende Tendenzen. Vatter sieht neben

2 Vgl. Adrian Vatter, Vom Extremtyp zum Normaltyp? Die Schweizerische Konsensusdemokratie im Wandel. Eine Re-Analyse von Lijpharts Studie für die Schweiz von 1997 bis 2007, in: Swiss Political Science Review, 14. Jg. (2008), H. 1, S. 1 - 47. Dieser Beitrag, von der Fritz-ThyssenStiftung als bester sozialwissenschaftlicher Aufsatz 2008 im deutschen Sprachraum ausgezeichnet, ist eine Art Kurzfassung des Bandes.

3 Vgl. ders., Kantonale Demokratien im Vergleich. Entstehungsgründe, Interaktionen und Wirkungen politischer Institutionen in den Schweizer Kantonen, Opladen 2002. 
der - wohl etwas überschätzten - Innovations- und der Integrationswirkung der Referenden ihre Schwächen in der betont minderheitenkritischen Sicht gegenüber „Fremdgruppen“ (zum Beispiel Verbot des Baus von Minaretten 2009). Ein Wort zur Terminologie: Der Rezensent ist nicht glücklich über den - verbreiteten - Begriff der „direkten Demokratie“, weil er suggeriert, eine repräsentative Demokratie sei nur eine indirekte, eine halbherzige.

Das wohl schwächste (nicht: schwache) Kapitel ist das zum Wahlsystem - Vatter unterteilt nach dem formalen Entscheidungs- und nicht nach dem Repräsentationsprinzip, das auf die Effekte zielt. So ordnet er Deutschland als „Mischsystem“ ein, und Länder mit Mehrmannwahlkreisen (wie Griechenland und Spanien), die mehrheitsbildend wirken, zu den Verhältniswahlsystemen. Wie die Abbildung zum Disproportionalitätsgrad (S. 87) verdeutlicht, ist dieser beim „Mischsystem“ in Deutschland erheblich geringer als bei der „Verhältniswahl“ in Griechenland. Dem Rezensenten war die erstaunlich hohe Zahl der Briefwähler (über 80 Prozent!) unbekannt.

Die Schweiz ist „eher freiheitlicher als gleicher, was auch ein Spiegelbild der politischen Kräfteverhältnisse mit einer im internationalen Vergleich starken bürgerlich-liberalen Mehrheit und einer eher schwachen Linken ist" (S. 563, Hervorhebung im Original). In der partiellen Erosion der konkordanzdemokratischen Elemente bei den politischen Eliten sieht der Verfasser wenig Gutes: Transparenz gehe auf Kosten von Konsens. Eine weitere Entfaltung konkurrenzdemokratischer Strukturen sei weder vorteilhaft noch wahrscheinlich. Der Autor betont wohl zu stark die Vorteile der Konkordanzdemokratie.

Vatter hat Linder nicht nur an der Universität Bern abgelöst, sondern auch dessen Werk als „die“ Studie zur Schweiz. Der Feind des Guten ist das Bessere. Die Systematik des Werkes, stärker ausgeprägt als bei Linder, besticht ebenso wie seine Faktentreue und seine politikwissenschaftliche Analysekraft, bezogen auf Methodik und Inhalt. Durch die strikte Orientierung am Kriterienkatalog Lijpharts ist die Studie auch für einen Ländervergleich reizvoll. Die Literaturkenntnis ist stupend, das Urteil eher zurückhaltend, wobei seine Präferenz für ein plebiszitäres Demokratieverständnis auffällt. Spricht der erste Satz des Buches von einer „allgemeinen Einführung in das politische System der Schweiz“ (S. 5), so lautet der letzte der Rezension dagegen: Die Studie vermittelt selbst dem Experten erhellende Erkenntnisse nicht nur zur helvetischen Demokratie im Wandel - „der Vatter“ ist „der neue Linder".

Eckhard Jesse

\section{Kleine Parlamente: welche Rolle spielt die Größe?}

Baldwin, Nicholas D.J. (Hrsg.): Legislatures of Small States. A Comparative Study (Series: Library of Legislative Studies), Routledge, London / New York 2013, 216 Seiten, £ 80,-.

Repräsentative Demokratie wird oftmals mit dem Hinweis auf die schiere Größe von Nationalstaaten begründet, die eine Demokratie nach antikem Vorbild verhindere. Ein Blick auf viele Staaten der Welt, die nur wenige Einwohner zählen, beweist allerdings, dass auch diese zu Repräsentationsmechanismen greifen. Der vorliegende, von Nicholas Baldwin herausgegebene Band interessiert sich vornehmlich und ganz allgemein für die Frage, ob die 